\title{
Retroperitoneoscopic approach for highly complex posterior renal hilar tumors
}

\author{
Jose Luis Bauza ${ }^{1}$, Valentí Tubau ${ }^{1}$, Jorge Guimerà ${ }^{1}$, Luis Ladaria ${ }^{1}$, Carlos Aliaga ${ }^{1}$, Pedro Piza ${ }^{1}$, Enrique \\ Pieras $^{1}$ \\ 1 Department of Urology, Hospital Universitario Son Espases, Palma de Mallorca, Illes Balears, \\ Spain
}

ABSTRACT

Objectives: To show our single-center experience in retroperitoneoscopic approach for highly complex posterior hilar tumors. Minimally invasive nephron sparing surgery for renal hilar tumors is extremely challenging due to their anatomic location, close to the main renal vessels and the collecting system (1). Transperitoneal approach is feasible, but highly complex because the anterior disposition of the vasculature. Retroperitoneal approach can easily provide access to the posterior hilar structures and the posterolateral surface of the kidney(2, 3).

Materials and Methods: We retrospectively reviewed our hilar renal tumor database and analyzed those in which a retroperitoneoscopic approach was chosen. The RENAL score was then calculated, and operative and ischemia times were recorded. We also collected the mean hospital stay and the presence of complications. Pathology reports and follow-up were also gathered.

Results: Five of our twelve highly complex hilar renal tumor patients were treated using a retroperitoneoscopic approach. Mean RENAL score was 10. Mean operative time was 135 minutes. Mean warm ischemia time was 14 minutes. Mean hospital stay was 4 days. We have recorded 2 complications. One patient required a transfusion and another presented with an urinary fistula which was treated by double J stent placement. The pathology report showed a clear cell renal cell carcinoma pT1a in most of the cases. Only one patient had a positive margin. To date, no recurrences have been noticed. Conclusions: The treatment of complex renal hilar tumors in a minimally invasive fashion is highly challenging even in experienced hands. Retroperitoneal partial nephrectomy is feasible, safe and effective for the treatment of such lesions. Long-term oncologic outcomes of this approach are awaited.

\section{CONFLICT OF INTEREST}

None declared.

\section{REFERENCES}

1. Sagalovich D, Dagenais J, Bertolo R, Garisto JD, Kaouk JH. Trifecta Outcomes in Renal Hilar Tumors: A Comparison Between Robotic and Open Partial Nephrectomy. J Endourol. 2018;32:831-6.

2. Fan X, Xu K, Lin T, Liu H, Yin Z, Dong W, et al. Comparison of transperitoneal and retroperitoneal laparoscopic nephrectomy for renal cell carcinoma: a systematic review and meta-analysis. BJU Int. 2013;111:611-21.

3. Kieran K, Montgomery JS, Daignault S, Roberts WW, Wolf JS Jr. Comparison of intraoperative parameters and perioperative complications of retroperitoneal and transperitoneal approaches to laparoscopic partial nephrectomy: support for a retroperitoneal approach in selected patients. J Endourol. 2007;21:754-9. 


\section{ARTICLE INFO}

\section{(iD) Jose Luis Bauza Quetglas}

https://orcid.org/0000-0002-8955-483X

Available at: : http://www.intbrazjurol.com.br/video-section/20190074_Bauza_et_al Int Braz J Urol. 2020; 46 (Video \#12): 485-6

Submitted for publication:

February 06, 2019

Accepted after revision:

January 27, 2020

Published as Ahead of Print:

February 10, 2020

Correspondence address:

Jose Luis Bauza Quetglas, MD

Department of Urology

Hospital Universitario Son Espases

79, Valldemossa Rd

Palma de Mallorca, 70120, Spain Telephone: + 34608 688-560

E-mail:peplluis15@hotmail.com 